\title{
A Method for Rehabilitation of Hand Dysfunction by Cyber Glove
}

\author{
Hao Chen ${ }^{1, a^{*}}$, Haining $\mathrm{Ou}^{2, b}$ and Hongxia Chen ${ }^{2, c}$ \\ ${ }^{1}$ School of Medical Information, Guangzhou University of Chinese Medicine, China \\ ${ }^{2}$ The Second Affiliated Hospital of Guangzhou University of Chinese Medicine, China \\ achenhao@gzucm.edu.cn, bliudonghui@gzucm.edu.cn, chxchen@gzucm.edu.cn
}

\begin{abstract}
Keywords: Rehabilitation Engineering, Hand Recovery Evaluation, Virtual Reality, Cyber Glove Abstract. This paper presents a new method of rehabilitation workstation for hand dysfunction. It has functions as follows: hand dysfunction diagnosis, assessment of disability, physical training. With the assistance of cyber gloves as well as hand strength training devices designed by the present authors, this workstation measures the hand joints movement accurately, and assesses and trains muscle strength. The system assists physiatrists to perform clinical activities such as disability evaluation, treatment decision, recovery evaluation, clinical information analysis and data filing. As a result, an integral system can be established for rehabilitation of hand dysfunction. This system makes the simultaneous treatments available to patients by simply increasing the number of hand-training devices; the efficiency of rehabilitation is improved consequently.
\end{abstract}

\section{Introduction}

Rehabilitation engineering is a combination of medicine and technology and also an important branch of biomedical engineering. As technology advances, revolutionary changes have taken place in rehabilitation's methods and devices, that is, it develops from the early simple mechanical apparatus of supporting or replacing human organs to the intelligence system of the active rehabilitation, training and evaluation [1].

Nowadays, in the products field of rehabilitation, many companies have already promoted a series of multi-functional rehabilitation equipments. For example: Hocoma, a Switzerland Company, has promoted an automatic robotic gait training and assessment system which is used for robotic treadmill training of patients with movement disorders. Multi-joint System 4, the product of Biodex USA, is a versatile ergometer that meets the demands of orthopedic rehabilitation, cardiac, sports medicine, wellness, or general conditioning program. The Compass of Proxomed Corp. made in Germany is designed for medical application in orthopedic and neuro-rehabilitation as well as in geriatric and sports medicine. All these products specializes the whole process from evaluation to training for the patients. In addition, some advanced technologies in other areas can be used for rehabilitation training, such as virtual reality technology(VR) and 3D motion image capture system with several image sensors. It is originally used for motion analysis and special effects of film or television, but also can be applied in the gait analysis [2].

Hands are most important organs for human beings. It has functions of self-care, labor, emotional expression, and exchange of ideas. Hand injuries are most common illnesses. Statistics show that more than one third is of hand injuries in a variety of trauma patients. Rehabilitation and reconstruction of hand function of patients play important roles to improve the quality of life. Researches suggest that early, reasonable, individualized and planned training schemes are effective for hand rehabilitation [3-5].

The premise of rehabilitation is evaluation, which includes muscle strength, motion of joints, sensory, flexibility, coordination, and etc. Proposed by Robert W. Lovett in 1916, Florence P. Kendall and other scholars supplemented and improved the Manual Muscle Test (MMT), which is widely used in the clinical evaluation of muscle strength [6-7]. Due to a variety of subjective factors and the lack of significant measurement limits of grading standards, MMT is still a semi-quantitative examination method. In addition, Dr. Eaton RG. first proposed Total Active Movement (TAM) to measure the activity of joints in 1975. TAM measures the ranges of metacarpophalangeal joint (MP), proximal interphalangeal joint (PIP) and distal interphalangeal joint (DIP) by using a protractor. 
TAM as a method of the tendon evaluation can fully reflect the situation of finger tendons and compare the active and passive activities of fingers before and after surgery. It is highly valuable but its measurement and calculation are complicated.

Overseas studies for hand rehabilitation have begun to introduce high-tech means such as intelligent sensing and measurement technology for objective functional assessment and rehabilitation. It is reported that a virtual reality-based system using a cyber glove and a MPII haptic glove to rehabilitate post-stroke patients in the chronic phase was developed at Rutgers University, NJ, USA. The two sensing gloves were integrated with VR system running on the PC workstation. The post-stroke patients interact with the system using the sensing gloves. Feedback was given on screen of the PC. Results showed all patients had significant improvement on their fingers' functions [8].

The rehabilitation of hand disorders generally includes a variety of conventional methods of physical therapy and occupational therapy in China . It has little difference from the international treatment concept [7], but is backward in terms of the devices of the treatment and assessment. Most clinical institutions still adopt the methods of using visual or goniometer to assess, because the execution of rehabilitation programs may not be under control.

In addition, due to the lack of automated equipment, physicians can only monitor the patients person to person in the treatments. When the number of patients increases, the occupational therapists or physicians get fatigue easily, and the work efficiency is lowered. Therefore, the development of hand rehabilitation workstation will make up for these shortcomings.

\section{System Architecture and Function}

\section{Hardware Design}

The hardware components of rehabilitation workstation include a personal computer, one pair of cyber gloves and 4-8 training devices. The system runs under control of custom-made software which contains a multimedia medical database management system. Other optional accessories include image scanner, printer, digital camera, and etc. The hardware system components are illustrated in figure 1.

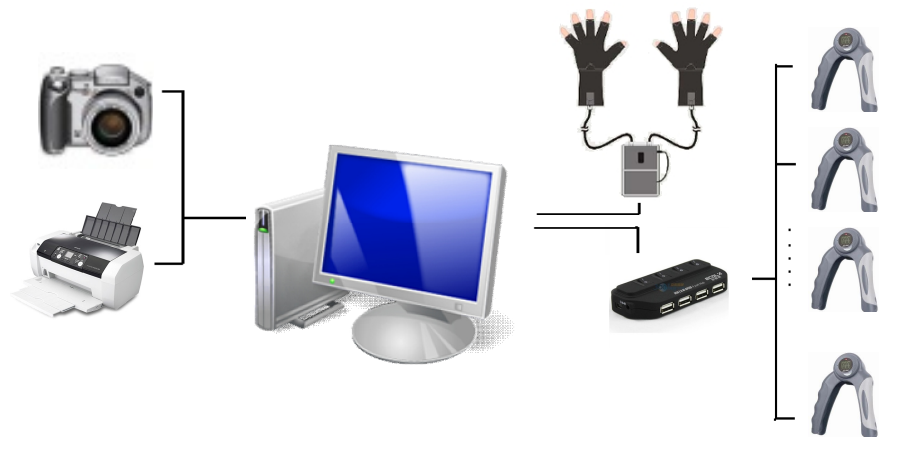

Figure 1. Hardware components of the workstation

A pair of 5DT Data Glove 5 Ultra with high- accuracy joint-angle measurements is selected here. The total weight of the glove together with data wire is about $150 \mathrm{~g}$. There are 5 sensors deployed on it, and the number of sensors is flexible. The gloves are stored with 1 bend sensors on each finger, 2 abduction sensors, plus 4 sensors measuring thumb crossover, palm arch, wrist flexion, and wrist abduction.

The 5DT CyberGlove systems come with the AD Box24, a microcontroller box which can host two cyber gloves with a total of sensor channels simultaneously. The Box has an USB connector for power supply and data transfer. 


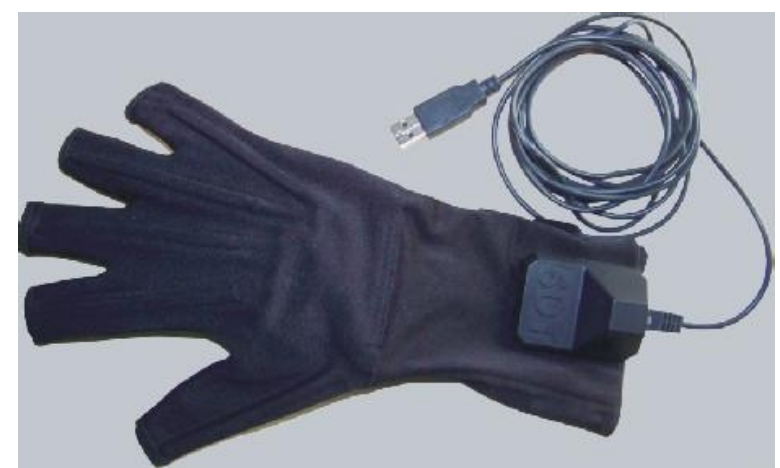

Figure 2. 5DT Data Glove 5 Ultra made by Fifth Dimension Technologies.

We added a hand strength testing and training apparatus into the workstation. In fact, it actually is a kind of enhanced muscle developer specially designed for patients. Comparing with ordinary developers the new one has extra functions as follows: grip calibration, counting and displaying, communication with PC, which means this gripper can adjust damping force in accordance with grip strength of patients. The operator can set a specific force to measure hand strength of the patient. Guided by the prescription written by the physician or occupational therapist, patients are trained according to the specified intensities and frequencies. All the information is acquired and displayed under the control of the multi-channel data acquisition box that specially designed for this system, and the data are uploaded to the PC host through USB connector in order to have real-time monitor of the rehabilitation quality.

At present, most of the hand training apparatus with simple damping adjustment in the market is specialized for the healthy people, and the damping force can not be exactly measured by the apparatus therefore can not be used for patients. Thus how to design a gripper which is simple, small and reliable to meet the functional needs mentioned above is one of the keys in this project.

\section{Software Design}

The system-oriented software of this workstation is written for system control and data acquisition and processing. According to its tasks, it is divided into 3 modules shown in figure 3.

The data acquisition module of data glove fulfills tasks of system initializing, calibration and data acquisition and filtering from specified signal channel.

The hand training module fulfills such tasks as damping force setting and measuring, hand training data monitoring, and etc.

The clinical information management system integrates personal information, disease condition, treatment prescription and training effect together with the data collected from above-mentioned modules to a database system which is used for inquiry, statistics and analysis. All the modules are controlled by the main program.

\section{Routines of Clinical Application}

After construction, the system can be applied to clinical practice, and the routines are as follows.

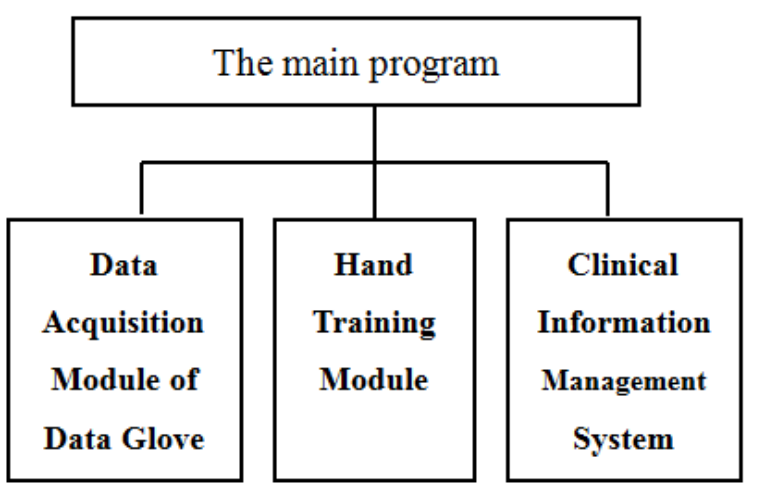

Figure 3. Software architecture of the workstation 
1. When a patient comes, the physician collects the patient's basic information by means of scanning, taking photos and etc. and establishes clinical record in the database.

2. The patient wears the data glove to test the movement ability of the joints of finger and its highest gripping force by gripper.

3. Referring to the results, the physician conducts the prescriptions and records them into database.

4. Monitored by the software, the patient does training. The physician can check and evaluate rehabilitation randomly and adjust the plan according to the curative effects. All these can be repeated until the end of the treatment.

5. After the treatment, the final clinical data of the patient is filed for later researches.

\section{Conclusion}

The research on new workstation for hand rehabilitation can not only help measure the movement trace of human hands quickly and accurately, test the muscle strength and train the hands, but also assist physicians to assess the disability degrees objectively and to decide personalized treatment plan. Moreover, the treatment plan can be enforced strictly under the real-time control of the system. Meanwhile, the clinical data can be analyzed and dealt with to provide the clinical assessment of different treatment stages so as to build the clinical database and do further scientific researches.

This system can make the treatments simultaneously available to many patients by simply increasing the number of devices, and correspondingly improve the efficiency of rehabilitation.

\section{Acknowledgment}

This research was supported by the Science and Technology Planning Project of Guangdong Province, China (Grant No.2011B031300015), and also supported by the National Natural Science Foundation of China (Grant No. J1310017)

\section{References}

[1] S.Jezernik, G.Colombo, T.Keller, et al, "Robotic Orthosis Lokomat: A Rehabilitation and Research Tool” , Neuromodulation, April 2003, 6(2):108-115.

[2] Rose FD, Attree EA, Johnson DA, “Virtual Reality: an Assistive Technology in Neurological Rehabilitation”, Curr Opin Neurol, 1996;9:461-467.

[3] Gao Curong, "Early Rehabilitation of Hand Injuries”, CHJR. of Rehabilitation. 1989, 4(1):5-7.

[4] Wu Weiqing, Yang Rong, et al, "Effects of Early Rehabilitation Training on Functional Recovery of Hand Injuries” , CHJR. of Rehabilitation 2006 21(4): 235-236.

[5] Liu Aihua, "Post-operative Retraining on Hand Flexor Tendon Injuries” , J. of Clinical Rehabilitative Tissue Engineering Research, 2002, 6(16): 2474.

[6] Wang Shuhuan, Hand Surgery, 2nd Edition, People's Medical Publishing House, Beijing, 2002.

[7] Wang Junhua, Introduction of Rehabilitation Medicine, People's Medical Publishing House, Beijing, 2010.

[8] R.Boian, A. Sharma, et al, “Virtual Reality-based Post-Stroke Hand Rehabilitation” , Proceedings of Medicine Meets Virtual Reality 2002 Conference, IOS Press, 64-70. 